\title{
Weichteilsarkom: längeres progressionsfreies Überleben, aber keine bessere Lebensqualität unter Pazopanib in der Zweitlinie
}

\begin{abstract}
Eine gute Lebensqualität zu erhalten, ist zentrales Ziel der Behandlung von Patienten mit fortgeschrittenem Weichteilsarkom. Die gesundheitsbezogene Lebensqualität war daher ein Endpunkt der PALETTE-Studie, in der die Wirksamkeit von Pazopanib in der Zweitlinie oder später untersucht wurde.
\end{abstract}

$\mathrm{B}$ ei fortgeschrittenen Weichteilsarkomen ist die Prognose ungünstig: Im metastasierten Stadium beträgt das mediane Gesamtüberleben ab Diagnose etwa 12 Monate. Die Suche nach aktiven Therapiestrategien zielt daher nicht nur darauf ab, progressionsfreies Überleben und Gesamtüberleben zu verlängern, sondern vor allem darauf, tumorbedingte Beschwerden zu kontrollieren, damit die Patienten bei guter Lebensqualität so lange wie möglich an den normalen Aktivitäten des täglichen Lebens teilhaben können. In Studien gilt daher besonderes Augenmerk den Beschwerden, die häufig Unabhängigkeit und Lebensqualität einschränken. Dazu zählen vor allem Fatigue, Schmerzen und Atemwegssymptome.

In der Phase-III-Studie PALETTE der EORTC (European Organization for research and treatment of cancer) waren 369 Patienten mit fortgeschrittenem Weichteilsarkom in der Zweitlinie oder in späteren Linien im Verhältnis 2:1 randomisiert entweder mit dem Multitarget-Tyrosinkinaseinhibitor Pazopanib (800 mg täglich, $\mathrm{n}=246$ ) oder Placebo $(\mathrm{n}=123)$ behandelt worden. Die Pazopanib-Therapie führte zu einem signifikant längeren progressionsfreien Überleben (median 4,6 vs. 1,6 Monate; Hazard Ratio 0,31; $<<0,001$ ). Allerdings litten die Patienten auch unter therapiebedingten Nebenwirkungen, vor allem Fatigue, Diarrhö, Nausea, Gewichtsverlust und Hypertonie.

Um den Einfluss der Pazopanib-Therapie auf die gesundheitsbezogene Lebensqualität (HRQoL) zu beurteilen, wurden zwei Instrumente herangezogen: der EORTC QLQ-C30-Fragebogen und der EQ-5D (EuroQol 5D). Basis für die vorliegende Auswertung waren die Ergebnisse des EORTC QLQ-C30-Fragebogens, die zu Beginn sowie nach 4, 8 und 12 Wochen erhoben wurden.

$94 \%$ der Patienten füllten den EORTC QLQ-C30 zu Beginn aus, nach 12 Wochen lag die Beteiligung bei $78 \%$ im Pla- cebo- und $81 \%$ im Pazopanib-Arm. Mittlerer und medianer HRQoL-Score waren zu Beginn in den beiden Therapiearmen vergleichbar hoch. Auch später waren keine signifikanten Unterschiede zwischen den Scores der beiden Therapiearme messbar, die vorab definierte Differenz von 10 Punkten wurde nie erreicht ( $p=0,291$; maximale Differenz: 3,8 Punkte). Unter Pazopanib gab es signifikant mehr Diarrhö $(\mathrm{p}<0,001)$, Appetitlosigkeit $(\mathrm{p}<0,001)$, Übelkeit und Erbrechen $(\mathrm{p}<0,001)$ sowie Fatigue $(p=0,012)$. In beiden Therapiearmen gingen die HRQoL-Scores mit der Zeit zurück.

Fazit: Die gesundheitsbezogene Lebensqualität wird beim fortgeschrittenen Weichteilsarkom unter Pazopanib trotz Verlängerung des PFS nicht verbessert. Allerdings verschlechtert sie sich trotz therapiebedingter Nebenwirkungen auch nicht. Kathrin von Kieseritzky

Coens $C$ et al. Health-related quality-of-life results from PALETTE: A randomized, double-blind, phase 3 trial of pazopanib versus placebo in patients with soft tissue sarcoma whose disease has progressed during or after prior chemotherapy-a European Organization for research and treatment of cancer soft tissue and bone sarcoma group global network study (EORTC 62072). Cancer. 2015;121(17):2933-41.

\section{Hier steht eine Anzeige.}

